\title{
Flux Tube Model Signals for Baryon Correlations in Heavy Ion Collisions
}

\author{
Apoorva Pate* \\ CHEP and SERC, Indian Institute of Science, Bangalore-560012, India
}

(Dated: June 21, 2018)

\begin{abstract}
The flux tube model offers a pictorial description of what happens during the deconfinement phase transition in QCD. The three-point vertices of a flux tube network lead to formation of baryons upon hadronization. Therefore, correlations in the baryon number distribution at the last scattering surface are related to the preceding pattern of the flux tube vertices in the quark-gluon plasma, and provide a signature of the nearby deconfinement phase transition. I discuss the nature of the expected signal, and how to extract it from the experimental data for heavy ion collisions at RHIC and LHC.
\end{abstract}

PACS numbers: $12.38 . \mathrm{Mh}, 25.75 . \mathrm{Nq}$

Keywords: Deconfinement, Flux tube model, Hadronization, Polyakov loop, Quark-gluon plasma, Cosmic microwave background radiation

\section{INTRODUCTION}

The theory of strong interactions, QCD, incorporates the non-perturbative phenomena of confinement and chiral symmetry breaking. These phenomena are expected to disappear at high temperatures and/or at large chemical potential, where QCD can be analysed reliably because its effective coupling becomes weak. How the phenomena arise in low temperature and low chemical potential region is crucial to our understanding of the QCD dynamics, ranging from hadronization in the early universe to the interior of neutron stars. A lot of effort has been devoted towards this goal, experimentally through the study of heavy ion collisions, theoretically through construction of phenomenological models (since we have not been able to solve QCD accurately), and computationally through simulations of lattice QCD. The results are often summarized as the phase structure of QCD in the temperature-chemical potential space. Recent reviews of the subject are available in Refs. [1 [ 6] .

Consider QCD with $N$ colors and $N_{f}$ degenerate quark flavors of mass $m$, at temperature $T$ and quark chemical potential $\mu$. The phase structure of this theory is depicted in Fig:1 in a schematic manner. (More details are needed to describe the real world QCD. Complexities arising from unequal quark masses and color superconductor phases at large chemical potential are omitted here.) Certain boundaries of the phase structure are better understood than the interior region, because of the exact symmetries present there. Explicitly:

(1) For $m=\infty$, the pure gauge theory has a finite temperature deconfinement phase transition, governed by the breaking of the global $Z_{N}$ center symmetry of the Polyakov loop. This transition is of first order for $N \geq 3$. (2) For $m=0=\mu$, the theory has a finite temperature chiral phase transition, governed by the restoration of

*Electronic address: adpatel@cts.iisc.ernet.in

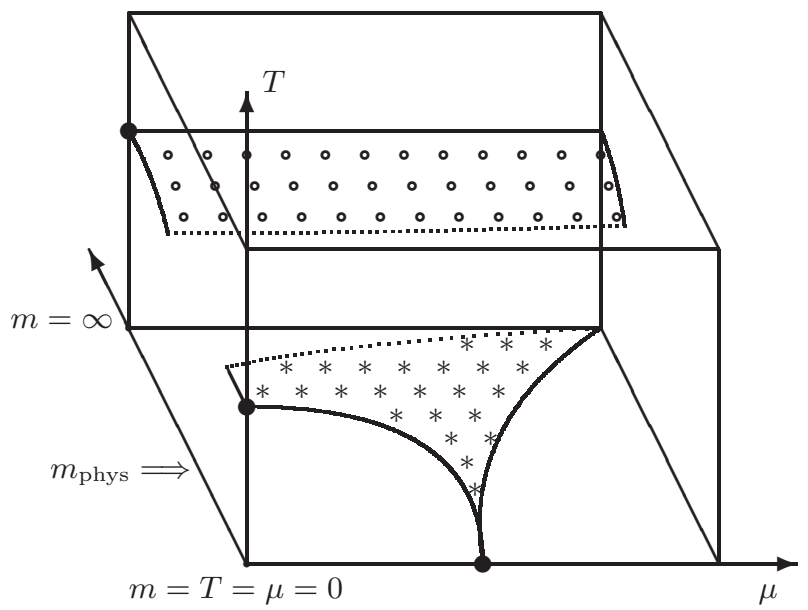

FIG. 1: Schematic description of the phase structure of QCD in the $m-T-\mu$ space. First-order transition surfaces are shown shaded, and critical lines are shown dotted. Value of $m$ corresponding to the real world QCD is indicated by an arrow. Color superconductor phases occurring at large chemical potential are omitted.

the flavor $S U\left(N_{f}\right)_{V}$ symmetry to $S U\left(N_{f}\right)_{L} \otimes S U\left(N_{f}\right)_{R}$. This transition is of first order for $N_{f} \geq 3$.

(3) For $m=0=T$, the theory has a baryon condensation phase transition, where the vacuum structure changes from $\langle\bar{\psi} \psi\rangle \neq 0$ to $\left\langle\psi^{\dagger} \psi\right\rangle \neq 0$. This transition occurs roughly when $\mu$ equals the constituent quark mass, and is also of first order.

First-order phase transitions are generically stable against small changes of symmetry breaking perturbations. So the above three phase transitions extend inwards, to varying extent, from the boundaries of the phase structure. The behavior expected from phenomenological models and numerical calculations is that the first-order transition surfaces end in critical lines, as shown in Fig 1] Specifically, for the physical values of the quark masses, there is no phase transition as $T$ is 
varied, unless $\mu$ is sufficiently large. In the analytic crossover region connecting high and low temperature regions, the three nearby transitions mentioned above cause various QCD properties to change rapidly. In general, which property is affected how much by which transition depends on the property concerned. To verify all the theoretical expectations, therefore, it is important to construct appropriate experimental observables that highlight the individual aspects of deconfinement, chiral symmetry restoration and baryon condensation, when QCD is tested at high temperature and/or at large chemical potential.

In this article, I focus on the signals in heavy ion collision experiments related to the deconfinement phase transition. The collisions produce a high energy nonequilibrium state of QCD matter, which subsequently expands and cools. The experimental signatures observed at RHIC and LHC demonstrate that the initial fireball first (quasi-)equilibrates to a quark-gluon plasma (QGP), which then hadronizes and decays. The detected quantities are multiplicities and distributions of various types of hadrons. Since a sizeable fraction of the colliding nuclei continues unscattered along the beam direction, the signals coming from the fireball are cleanly observable only in sufficiently transverse directions, say for pseudorapidity $|\eta|<\eta_{\max }$. These signals can be broadly separated into two types: (a) those describing the initial non-equilibrium fireball state and its approach to equilibrium, and (b) those characterizing the properties of an equilibrated QGP. The former consist of particles that can manage to pass through the QGP, e.g. direct photons and leptons, heavy quark jets, high $p_{T}$ jets and the elliptic flow. The latter consist of distributions of moderate $p_{T}$ hadrons produced close to the surface of the fireball, and they are the ones I want to relate to properties of the QGP.

The QGP is a strongly interacting medium, while the hadrons resulting from its decay do not interact much with each other. The hadronization process thus goes through a stage where the scattering events among the components emerging from the fireball drop rapidly. Afterwards, the hadrons essentially propagate radially outward without scattering, although the unstable ones decay. From the observed detector signals, it is possible to backtrack the distributions of hadrons to this "kinetic freeze-out" stage of the fireball. This stage is analogous to the "last scattering surface" in the evolution of the cosmic microwave background radiation (CMBR), and several methodologies developed to study the fluctuations there are useful in its analysis.

It should be kept in mind that the QGP is strongly coupled in the cross-over region, and the decoupling of hadrons goes through several stages instead of being instantaneous [7]. Hadronization of the (quasi)equilibrated QGP takes place at energy density $\epsilon_{\mathrm{cr}} \simeq$ $1 \mathrm{GeV} / \mathrm{fm}^{3}$ and $T_{\text {cr }} \simeq 175 \mathrm{MeV}$. Shortly thereafter, inelastic scattering of hadrons stops, resulting in "chemical freeze-out" when $T_{\text {chem }} \simeq 170 \mathrm{MeV}$. Upon further expan- sion, elastic and resonant scattering (mediated largely by pions) ceases, producing "kinetic freeze-out" when $T_{\text {kin }} \simeq 120 \mathrm{MeV}$. The scatterings contribute significantly to the thermalization of the hadron momenta, but assuming that the hadronic medium has low diffusion, we can see through them to the patterns in the QGP.

The distribution of hadrons at the kinetic freeze-out stage has often been modeled as a thermalized hadron resonance gas. Such thermal models fit the observed multiplicities of various hadrons, but miss all the multiparticle correlations between hadrons. The strongly interacting QGP should have many correlations, and more accurate models are therefore needed to relate them to observable signals. In what follows, I describe how a flux tube model providing a physical picture of the deconfinement process predicts specific two-particle correlations in the distribution of hadrons, going beyond the single particle multiplicities. I first present a summary of the model, and then discuss the baryon number correlations predicted by it.

\section{THE FLUX TUBE MODEL [8]}

\section{A. Phenomenology}

The flux tube model of QCD is motivated by the dual superconductor description of linear color confinement [9], where condensation of color magnetic charges restricts color-electric fields to vortex-like configurations. Although an exact derivation of the model from QCD has not been found, the model describes strong coupling expansions in lattice QCD and has been phenomenologically quite successful. A characteristic property of the flux tube is its energy per unit length, i.e. the string tension $\sigma$. Other than that, the flux tube has a finite width $w$ and a persistence length $a$ (arising from the tube's stiffness so that the flux tube has to go a certain distance before it can freely reorient itself), both assumed to be of order $\Lambda_{Q C D}^{-1}$.

The flux tubes have to obey the constraint of Gauss's law. So they terminate only on quarks, and interact only at $N$-point vertices. These two features represent the invariant tensors $\delta_{a b}$ and $\epsilon_{a b c}$ (in case of $S U(3)$ ) used to describe the meson and the baryon wavefunctions. Other multi-quark hadron states are phenomenologically not prominent, except for multi-nucleon nuclei, and so all other interactions among the flux tubes are ignored in the model [10]. Note that a glueball would be represented by a closed flux tube loop in this description.

The finite temperature behavior of the model is governed by the competition between the energy and the entropy of the flux tube configurations. At low temperatures, energy wins, keeping down the total length of the flux tubes. At high temperatures, entropy dominates, producing elaborate structures of the flux tubes all over the space.

First consider the situation for the pure gauge the- 


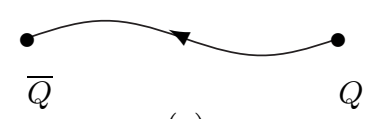

(a)

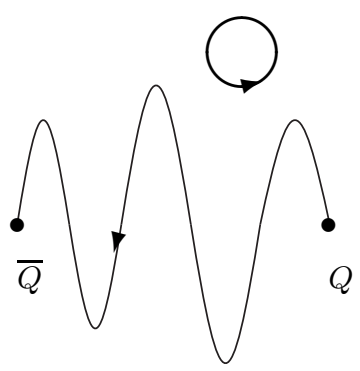

(b)

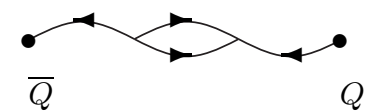

(c)

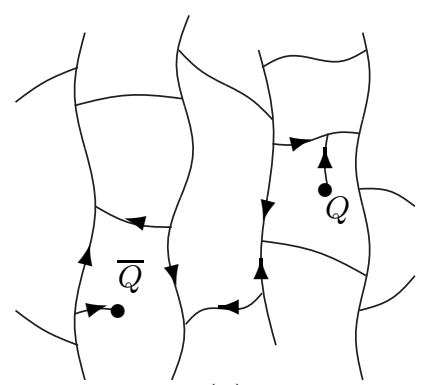

(d)
FIG. 2: Possible flux tube configurations connecting a static quark-antiquark pair, as the temperature is increased (from top to bottom), and when baryonic vertices are included (from left to right).

ory with only static color sources, i.e. $m=\infty$. As the temperature is increased the flux tubes oscillate more, and also produce more vertices. Some of the possible configurations are shown in Fig 2] In absence of vertices, corresponding to the $S U(2)$ gauge theory, there is a second-order deconfinement phase transition when the flux tube length diverges and the quark-antiquark pair loses information about each-other's position. In presence of vertices, corresponding to $S U(N>2)$ gauge theories, the flux tubes can percolate the space in a network before their length can diverge. That also allows the quark-antiquark pair (hooked on to the network) to lose information about each-other's position, but produces a first-order deconfinement phase transition.

When finite mass quarks are included in the model, they can break the flux tubes by quark-antiquark pair production from the vacuum. Baryon number production at finite chemical potential can also break the flux tubes. These possibilities are illustrated in Fig 3. With the flux tube network breaking up, the strength of the deconfinement phase transition weakens as the quark mass is lowered from $m=\infty$ and/or as the chemical potential is increased. Numerical estimates show that for $N_{f}=3$ QCD at small chemical potentials, the first-order deconfinement phase transition ends in a critical line around $m=1.5 \mathrm{GeV}$, as depicted in Fig 1 Although the crossover region does not have any sharp behavior of the deconfinement phase transition, we can still investigate whether the percolating flux tube scenario suggests any detectable signal there.

\section{B. Quantitative Formulation}

It is straightforward to formulate the model on a lattice with spacing $a$. The flux tubes live on the links, while the quarks and vertices live on the sites. The variables (a)

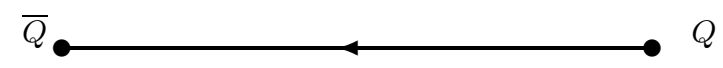

(b)

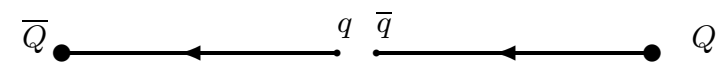

(c)

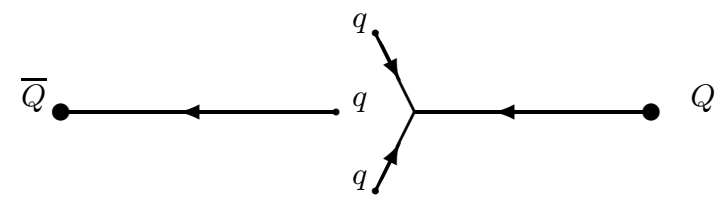

FIG. 3: A color-electric flux tube can break when dynamical quarks are included in the theory. (a) A flux tube produced by static color sources. (b) Its breaking by a quark-antiquark pair appearing from the vacuum. (c) Its breaking by a baryon appearing from the vacuum at finite chemical potential.

take values $0, \pm 1$, depending on the direction of the flux (for $N>2$ ), as shown in Fig 4 The total energy of a flux tube configuration is

$$
E=\sigma a \sum_{i, \mu}\left|n_{i, \mu}\right|+m \sum_{i, f}\left|p_{i, f}\right|+v \sum_{i}\left|q_{i}\right|,
$$

where $v$ denotes the energy cost of an $N$-point vertex, and $f$ sums over the $2 N_{f}$ spin and flavor quark degrees of freedom. For a cubic lattice, the constraint of Gauss's law at every site becomes

$$
\sum_{\mu}\left(n_{i, \mu}-n_{i-\mu, \mu}\right)-\sum_{f} p_{i, f}+N q_{i} \equiv \alpha_{i}=0 .
$$

When Gauss's law is applied globally, the contribution of the flux variables $n_{i, \mu}$ cancels. We then have two ways of determining the total baryon number $B$, either from the quark variables $p_{i, f}$ or from the vertex variables $q_{i}$ :

$$
\begin{aligned}
& \sum_{i} \alpha_{i}=N \sum_{i} q_{i}-\sum_{i, f} p_{i, f}=0 \\
& \Longrightarrow B=\frac{1}{N} \sum_{i, f} p_{i, f}=\sum_{i} q_{i} .
\end{aligned}
$$
is

The grand canonical partition function for the system

$$
Z[T, \mu]=\sum_{n_{i, \mu}, p_{i, f}, q_{i}} \exp \left[-\frac{E-\mu N B}{T}\right] \prod_{i} \delta_{\alpha_{i}, 0},
$$
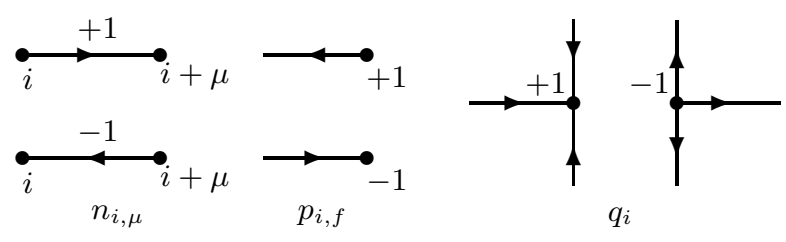

FIG. 4: The link and site variables for the flux tube model. 
with Eq.3 implying that quark chemical potential $\mu$ is equivalent to vertex chemical potential $N \mu$. The grand canonical partition function is fully factorized by expressing the Kronecker delta constraint at every site as

$$
\delta_{\alpha_{i}, 0}=\int_{-\pi}^{\pi} \frac{d \theta_{i}}{2 \pi} e^{i \alpha_{i} \theta_{i}} .
$$

The sum over the variables $n_{i, \mu}, p_{i, f}, q_{i}$ can then be carried out explicitly, resulting in

$$
\begin{aligned}
Z[T, \mu] & =\int_{-\pi}^{\pi} \prod_{i} \frac{d \theta_{i}}{2 \pi} \prod_{i, \mu}\left(1+2 e^{-\sigma a / T} \cos \left(\theta_{i+\mu}-\theta_{i}\right)\right) \\
& \times \prod_{i}\left(1+2 e^{-m / T} \cos \left(\theta_{i}+i \frac{\mu}{T}\right)\right)^{2 N_{f}} \\
& \times \prod_{i}\left(1+2 e^{-v / T} \cos \left(N \theta_{i}\right)\right) .
\end{aligned}
$$

The equivalence of quark and vertex chemical potentials, following from Eq.(3), makes $Z[T, \mu]$ invariant under the global symmetry transformation, $\theta_{i} \rightarrow \theta_{i}+\epsilon$, for any complex value of $\epsilon$.

By extending the allowed values of the variables $n_{i, \mu}, p_{i, f}, q_{i}$ to all integers [11], the grand canonical partition function can be converted to 12 ]

$$
\begin{aligned}
Z[T, \mu] & =\int_{-\pi}^{\pi} \prod_{i} \frac{d \theta_{i}}{2 \pi} \exp \left[J \sum_{i, \mu} \cos \left(\theta_{i+\mu}-\theta_{i}\right)\right. \\
& \left.+h \sum_{i} \cos \left(\theta_{i}+i \frac{\mu}{T}\right)+p \sum_{i} \cos \left(N \theta_{i}\right)\right]
\end{aligned}
$$

The systems represented by Eq.(6) and Eq.(7) are both in the universality class of the XY spin model in the presence of an ordinary magnetic field as well as a $Z(N)$ symmetric magnetic field. Their phase structures are similar, and their couplings are related by

$$
J=2 e^{-\sigma a / T}, \quad h=4 N_{f} e^{-m / T}, \quad p=2 e^{-v / T} .
$$

In quantitative analysis, Eq.(17) has the convenience that its algebraic structure is suitable for treatment using wellestablished methods in statistical mechanics.

The physical meaning of the site variables $\theta_{i}$ can be uncovered by looking at the free energy of a static color charge in the system. Introduction of a quark at site $j$ modifies the Gauss's law constraint there as $\delta_{\alpha_{j}, 0} \rightarrow$ $\delta_{\alpha_{j},-1}$. That makes the free energy of a static quark

$$
\exp \left(-F_{q} / T\right)=\left\langle\exp \left(-i \theta_{j}\right)\right\rangle
$$

In the finite temperature gauge field theory language, this free energy is given by the expectation value of the Polyakov loop $P_{j}$ at the site $j$. So we arrive at the correspondence that $\theta_{i}$ represents the phase of the Polyakov loop $P_{i}$, and the flux tube description of deconfinement is dual to the familiar Polyakov loop description of deconfinement [13]. The important advantage of the flux tube description is that it provides a position space visual representation of what happens as the temperature is varied in QCD.

Another advantage of the flux tube formulation is that it can be numerically simulated without any fermion sign problem at finite chemical potential [14 17], since Eq.(4) involves only real positive weights while Eq.(7) necessitates complex weights.

\section{Limitations}

The flux tube model described above does not incorporate the chiral nature of the quark degrees of freedom. So it cannot properly address the features in the QGP crossover region that can be considered consequences of the nearby chiral phase transition. Attempts have been made to combine Polyakov loop and Nambu-Jona-Lasinio models [18 20], to study chiral properties of the QGP. They have treated the Polyakov loop only as a mean field without its full dynamics, but have still provided reasonable fits to static QGP properties extracted from lattice QCD simulations. They need to be extended by including the kinetic term for the Polyakov loop dynamics, e.g. $J \neq 0$ in Eq.(7), to obtain a good estimate of the fluctuations. One of the predictions of chiral symmetry restoration at high temperature is an increased production of baryons relative to mesons, due to a decrease in the constituent quark mass. The same enhancement is also predicted by the increase in the number of flux tube vertices at high temperature. This coincidence makes the prediction robust, and it has indeed been observed [21], but an estimate of its magnitude requires accurate treatment of both confinement and chiral dynamics. That is an exercise for the future.

Without the chiral properties of quarks, the flux tube model is also unable to say much about the baryon condensation phase transition. On the other hand, influence of a finite chemical potential on the deconfinement phase transition can be inferred from the behavior of the grand canonical partition function. The particle $\leftrightarrow$ antiparticle symmetry of QCD corresponds to invariance of $Z[T, \mu]$ under simultaneous sign flips of the variables $n_{i, \mu}, p_{i, f}, q_{i}$. The partition function is therefore an even function of $\mu$, i.e. $Z[T, \mu]=Z[T,-\mu]$. Convexity of the exponential function then implies

$$
Z[T, \mu] \geq Z[T, \mu=0], \quad F[T, \mu] \leq F[T, \mu=0] .
$$

$F[T, \mu]$ is continuous across the coexistence surface, and $d F=-S d T-N d \mu$ at constant volume. So for positive latent heat, it follows that introduction of a chemical potential decreases the transition temperature.

Furthermore, as described earlier, a non-zero chemical potential weakens the signal from the deconfinement phase transition. On the other hand, it enhances the signal from the baryon condensation phase transition, observable in baryon number susceptibilities. Baryon condensation needs to be treated as a site percolation prob- 
lem and not as bond percolation by flux tubes. That will not be addressed here.

\section{BARYON NUMBER CORRELATION SIGNALS}

Let us consider the flux tube scenario of what happens in the heavy ion collision experiments as the fireball of the QGP expands and cools. Even though the crossover region relevant to experiments does not possess a single percolating flux tube network, it can still contain many finite clusters of flux tubes. Because the flux is directed, an obvious feature of every such cluster is that any neighbor of a vertex is an anti-vertex and vice versa (see Fig.2(d)). During the evolution of the fireball, the total baryon number is conserved 22], and so the vertices can only be locally pair-produced or pair-annihilated. As the QGP hadronizes, the flux tube clusters start breaking up. After the chemical freeze-out stage, there is no more production or annihilation of vertices; every vertex ends up in a baryon and every anti-vertex ends up in an antibaryon. In the absence of subsequent largescale diffusion, the radial propagation of (anti)baryons preserves the geometric pattern of (anti)vertices present at the chemical freeze-out stage. As a result, the angular positions of the (anti)baryons seen in the detector can be backtracked to the angular positions of the (anti)vertices on the surface of the fireball at the chemical freeze-out stage. This pattern of vertices on the surface of the fireball can then be analysed for correlations and fluctuations, using techniques similar to those used to analyse the temperature fluctuations in the cosmic microwave background radiation [23].

The above description is a simple consequence of picking the right variables to visualize confinement dynamics in QCD. Although quantitative estimates of the (anti)vertex distributions would require numerical simulations, there are certain qualitative features that can be gleaned with much less effort. The fact that heavy ion collisions produce a sizeable number of antibaryons [21], from an initial state that has none, means that (a) a good number of baryonic and antibaryonic vertices are produced in the fireball, and (b) fragmentation of flux tubes during hadronization is more likely than their shrinking and coalescing that would annihilate vertices with antivertices. Our aim is to look for a specific pattern in the distribution of the produced (anti)vertices. Note that the flux tube scenario is unable to say anything regarding the energy and the momenta of the hadrons that emerge from the fireball.

\section{A. Experimental Data Parametrization}

There are several differences in the type of data gathered from CMBR and from heavy ion collision experiments, which need to be kept in mind when studying the two with similar techniques.

(1) The CMBR is a single high statistics event where homogeneity and isotropy of the universe allow accurate determination of the distribution parameters, while heavy ion collisions are multiple modest statistics events where ensemble averaging improves the accuracy of the distribution parameters.

(2) The temperature data of CMBR are real numbers, while the baryon number data of heavy ion collisions are integers that need to be binned and smeared.

(3) The average temperature and size of the "last scattering surface" are well-determined for CMBR, while the number of participating nucleons and the fireball volume fluctuate considerably in case of heavy ion collisions and are not accurately determined.

(4) The CMBR data cover the full $4 \pi$ solid angle and hence can be easily parametrized using the orthogonal basis of the spherical harmonics, while the heavy ion collision data are restricted to sufficiently transverse directions only and a suitable orthogonal basis for its parametrization has to be found.

Let us assume that the available data $\operatorname{cover} \theta_{m}<\theta<$ $\pi-\theta_{m}$, and label the angular distribution by the unit vector $\hat{n}$. The heavy ion collision experiments have an axial symmetry around the beam axis as well as the reflection (or parity) symmetry $\theta \leftrightarrow \pi-\theta$. These symmetries are sufficient to prevent the average baryon number flow in any direction, and can be used to improve the statistical accuracy of the correlations in the data. Fourier expansion helps in search for correlations by orthogonal separation of scales, and the baryon number distribution can be partially expanded as

$$
b(\hat{n}) \equiv b(\theta, \phi)=\frac{1}{\sqrt{2 \pi}} \sum_{\sigma= \pm} \sum_{m=-\infty}^{\infty} b_{m}^{\sigma}(\theta) e^{i m \phi} .
$$

Here $b_{m}^{ \pm}(\theta)= \pm b_{m}^{ \pm}(\pi-\theta)$, reality of $b(\hat{n})$ implies $b_{m}^{\sigma *}(\theta)=$ $b_{-m}^{\sigma}(\theta)$, and $\langle b(\hat{n})\rangle=b_{0}^{+}(\theta) / \sqrt{2 \pi}$. In a sense, the positive and the negative parity terms correspond to all the even and all the odd values of $l$, respectively, of the spherical harmonics expansion. Since the hadrons emerging from the fireball in longitudinal directions go undetected, the total and detected baryon numbers of a collision event are (note that $\tanh \eta=\cos \theta)$ :

$$
\begin{aligned}
& B_{\mathrm{tot}}=\sqrt{2 \pi} \int_{-1}^{1} d(\cos \theta) b_{0}^{+}(\theta), \\
& B_{\mathrm{det}}=\sqrt{2 \pi} \int_{-\cos \theta_{m}}^{\cos \theta_{m}} d(\cos \theta) b_{0}^{+}(\theta) .
\end{aligned}
$$

The two-point baryon number correlation function is the ensemble average $\left\langle b(\hat{n}) b\left(\hat{n}^{\prime}\right)\right\rangle$. Because of the axial symmetry, it depends only on the difference of the azimuth angles $\phi-\phi^{\prime}$, and because of the reflection symmetry the product of the parities $\sigma \sigma^{\prime}$ has to be one. Therefore,

$$
\left\langle b_{m}^{\sigma}(\theta) b_{-m^{\prime}}^{\sigma^{\prime}}\left(\theta^{\prime}\right)\right\rangle=\left\langle b_{m}^{\sigma}(\theta) b_{m^{\prime}}^{\sigma^{\prime} *}\left(\theta^{\prime}\right)\right\rangle=\delta_{\sigma \sigma^{\prime}} \delta_{m m^{\prime}} C_{m}^{\sigma}\left(\theta, \theta^{\prime}\right),
$$


and the two-point correlation function becomes

$$
\left\langle b(\hat{n}) b\left(\hat{n}^{\prime}\right)\right\rangle=\frac{1}{2 \pi} \sum_{\sigma= \pm} \sum_{m=-\infty}^{\infty} C_{m}^{\sigma}\left(\theta, \theta^{\prime}\right) e^{i m\left(\phi-\phi^{\prime}\right)} .
$$

Inverting this relation, we obtain

$$
\begin{aligned}
C_{m}^{\sigma}\left(\theta, \theta^{\prime}\right) & =\frac{1}{2 \pi} \int_{0}^{2 \pi} d \phi \int_{0}^{2 \pi} d \phi^{\prime} e^{i m\left(\phi^{\prime}-\phi\right)}\left\langle b^{\sigma}(\hat{n}) b^{\sigma}\left(\hat{n}^{\prime}\right)\right\rangle, \\
b^{ \pm}(\hat{n}) & =\frac{1}{2}(b(\theta, \phi) \pm b(\pi-\theta, \phi)) .
\end{aligned}
$$

The coefficient functions $C_{m}^{ \pm}\left(\theta, \theta^{\prime}\right)$ are real and symmetric. They contain all the information about the twopoint correlation function. The non-trivial correlations are given by the connected contributions,

$$
\begin{gathered}
\left\langle b(\hat{n}) b\left(\hat{n}^{\prime}\right)\right\rangle_{c}=\left\langle b(\hat{n}) b\left(\hat{n}^{\prime}\right)\right\rangle-\langle b(\hat{n})\rangle\left\langle b\left(\hat{n}^{\prime}\right)\right\rangle, \\
{\left[C_{m}^{\sigma}\left(\theta, \theta^{\prime}\right)\right]_{c}=C_{m}^{\sigma}\left(\theta, \theta^{\prime}\right)-\delta_{m 0} \delta_{\sigma+} b_{0}^{+}(\theta) b_{0}^{+}\left(\theta^{\prime}\right) .}
\end{gathered}
$$

In practice, depending upon the resolution available in the data, (a) the expansion can be truncated at a suitable value of $|m|$, and (b) the $\theta$-dependence can be further subdivided into smaller bins.

Similar parametrizations of the data can be carried out for distributions of other conserved quantities also, e.g. the electric charge or the strangeness. As a matter of fact, experimentally observed correlations for different conserved quantities can be compared with each other, to illustrate different aspects of the QGP dynamics. The flux tube model predictions are the cleanest in case of the baryon number, and illustrate the deconfinement mechanism. Different models with appropriate features would be required to understand the dynamics of other conserved quantities.

It should be noted that the theoretical estimates of the correlations are obtained assuming that the volume of the QGP is sufficiently large. On the other hand, the number of participating nucleons in the collisions, and hence the volume of the fireball, depends on the centrality of the heavy ion collisions. So to determine as well as to control the consequent systematic effects, it is necessary to determine the correlation functions in different centrality ranges separately.

\section{B. Theoretical Expectations}

Theoretically, the equilibrium correlations between flux tube vertices are straightforward to calculate in the three-dimensional position space, which can then be projected onto the observed surface of the fireball. An alternating neighbor pattern of vertices and anti-vertices, expected in a percolating flux tube network, would give rise to two-point baryon number correlations similar to the two-point charge correlations in ionic liquids (with ions of comparable size) such as $\mathrm{Cs}^{+} \mathrm{Cl}^{-}$. The key common ingredient in the two cases is the hard-core repulsion between the objects involved.

For discrete objects located at $\vec{r}_{i}$, a convenient description of the position space correlations is in terms of the pair distribution function

$$
\rho(\vec{r}) g\left(\vec{r}, \vec{r}^{\prime}\right) \rho\left(\vec{r}^{\prime}\right)=\left\langle\sum_{i \neq j} \delta\left(\vec{r}-\vec{r}_{i}\right) \delta\left(\vec{r}^{\prime}-\vec{r}_{j}\right)\right\rangle,
$$

where $\rho(\vec{r})=\left\langle\sum_{i} \delta\left(\vec{r}-\vec{r}_{i}\right)\right\rangle$ is the local density of the objects. In homogeneous and isotropic fluids, $\rho$ is independent of the position and $g$ depends only on $\left|\vec{r}-\vec{r}^{\prime}\right|$. Choosing $\vec{r}^{\prime}=0$, then we have

$$
\rho g(r)=\left\langle\sum_{i \neq 0} \delta\left(\vec{r}-\vec{r}_{i}\right)\right\rangle,
$$

Interactions fade away at long distances, and so asymptotically $g(r \rightarrow \infty)=1$. For objects with no correlations, e.g. an ideal gas, $g(r)=1$. For objects with hard-core repulsion, $g(0)=0$, and beyond the hard core $g(r)$ tends to its asymptotic value exhibiting damped oscillations [24], as illustrated in Fig 5. In particular, the distance scale of the oscillations is determined by the inter-object separation (the first peak corresponds to the likely nearestneighbor separation, the second peak corresponds to the likely next-nearest-neighbor separation, and so on), and the amplitude of the oscillations is determined by how tightly the objects are packed together.

To detect the correlations between vertices and antivertices, it is useful to compare the pair distribution functions for $|q(\vec{r})|\left|q\left(\vec{r}^{\prime}\right)\right|$ and $q(\vec{r}) q\left(\vec{r}^{\prime}\right), g_{|v|}$ and $g_{v}$ respectively, on the same data sets. Comparison of $g_{|v|}$ and $g_{v}$ keeps under control the effects arising from variation in the number of participating nucleons in the heavy ion collisions. Both follow the form of Eq.(19), with the sum running over vertices and anti-vertices. The former omits any vertex value signs and uses the density $\rho_{|v|}$, while the latter includes the vertex values, i.e. $q_{i} q_{j}$ on the righthand side of Eq.(19) and $q_{i}$ in obtaining the density $\rho_{v}$ (note that $\rho_{v}<\rho_{|v|}$ ).

For a percolating flux tube network, the function $g_{|v|}(r)$ should behave similar to that for hard-core objects, with the nearest-neighbor separation of the order of the baryon size. Similar behavior would be expected from several other fluid models also, so $g_{|v|}(r)$ can be treated as a model-insensitive reference function. In contrast, in a percolating flux tube network, successive neighbors contribute with opposite signs to $g_{v}(r)$. The resultant pair distribution function would then have oscillations of the form sketched in Fig 5 . For a system with little or no correlations between vertices, e.g. single particle thermal distribution models, the probability of occurrence of a vertex or an anti-vertex at any location is proportional to its overall density. Contributions to the right-hand side of Eq.(19) would then have the same density factors as on the left-hand side, and $g_{v}(r)$ would behave the same 


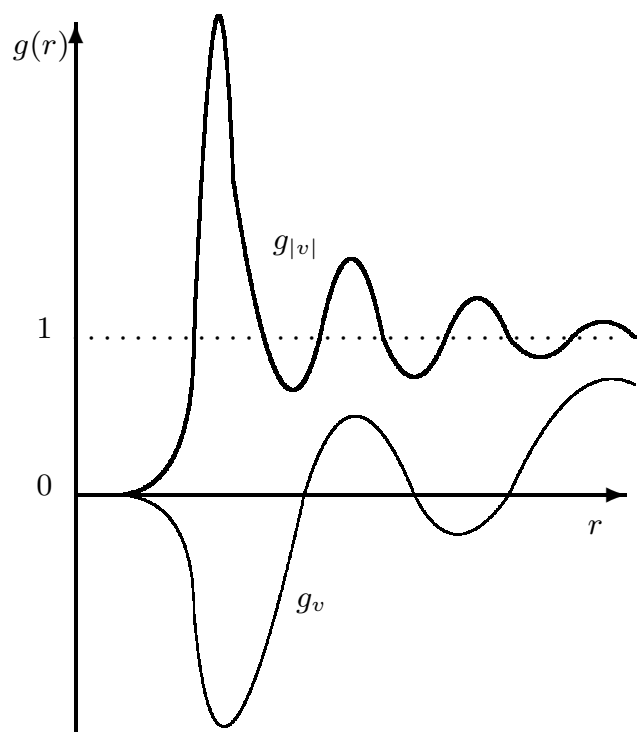

FIG. 5: Schematic representation of the pair distribution functions $g_{|v|}(r)$ (thick line) and $g_{v}(r)$ (thin line). The former is similar to that for objects with hard-core repulsion. The latter is for a percolating flux tube network where vertices and anti-vertices alternate.

way as $g_{|v|}(r)$. Thus $g_{v}(r)$ is sensitive to the correlations present between vertices and anti-vertices, and the contrast between $g_{|v|}(r)$ and $g_{v}(r)$ can be used as a measure of these correlations.

The percolating flux tube network in the high temperature QGP is maximally connected for $m=\infty$, with $N$ nearest neighbors for each vertex and anti-vertex. The network starts breaking up as the quark mass is lowered, more so if the chemical potential is also increased. Even individual baryons that are not part of any flux tube cluster can appear. Such a break up reduces the correlations between neighbors, smoothening the oscillations of the pair distribution function, analogous to what happens when a liquid is heated up. For the physical values of the quark masses, there is no deconfinement phase transition and no single percolating flux tube network is expected in the QGP. Still, if large enough flux tube clusters survive in the cross-over region, they would contribute to the contrast between $g_{v}(r)$ and $g_{|v|}(r)$. The strength of the contrast, therefore, measures the extent to which the nearby deconfinement phase transition influences the properties of the QGP in the cross-over region.

In general, the first peak of the pair distribution function is the most informative about the fluid properties. Its location provides an estimate of the inter-object separation, and the area under it is a measure of the number of nearest neighbors. In the present case, it is also the place where the contrast between $g_{v}(r)$ and $g_{|v|}(r)$ is the maximum, and so it is the best feature for identifying the correlations between vertices and anti-vertices. Note that for specific values of the parameters in Eq.(8), numerical simulations of the flux tube model can obtain the functions $g_{v}(r)$ and $g_{|v|}(r)$ for an equilibrated homogeneous and isotropic QGP.

\section{Connecting Theory to Experiment}

Projection of the three-dimensional pair distribution function onto the surface of the fireball smears the oscillatory structure of $g(r)$. The resultant angular distribution can be expressed in terms of a smearing function as

$$
w(\alpha)=\int_{r_{\min }}^{r_{\max }} S(\alpha, r) g(r) d r, \quad \int_{0}^{\pi} S(\alpha, r) d \alpha=1 .
$$

Here the integral over $r$ has to be sufficiently restricted so as not to lose the oscillatory signal. Since the correlations that we are interested in are of short range, it is convenient to remove the uniform part of the distribution function, e.g. look at $g(r)-1$ which describes the connected part. In the context of picking up the vertex correlation signal, it is even better to look at the difference $g_{v}(r)-g_{|v|}(r)$, which vanishes for both $r \rightarrow 0$ and $r \rightarrow \infty$.

In a specific setting, the smearing can be performed with sufficient accuracy numerically. Approximate analytic result can be obtained, nevertheless, under the assumption that the inter-vertex separation $r$ is much smaller than the radius of the fireball $R$. Let $\vec{a}$ be the position of the center of the pair with respect to the center of the fireball, and let $\beta$ be the angle between $\vec{a}$ and $\vec{r}$. Then the pair separation $r$ projects to angular separation $\alpha=(r / a) \sin \beta$. Taking expectation value over $\beta$ and $a$, for a homogeneous and isotropic distribution of pairs in the fireball, the smearing function becomes:

$$
\begin{aligned}
S(\alpha, r) & =\int_{0}^{R} \frac{3 a^{2} d a}{R^{3}} \int_{0}^{\pi} \frac{\sin \beta d \beta}{2} \delta\left(\alpha-\frac{r}{a} \sin \beta\right) \\
& =\int_{0}^{R} \frac{3 a^{2} d a}{R^{3}} \frac{\alpha a^{2}}{r \sqrt{r^{2}-\alpha^{2} a^{2}}} \\
& =\left\{\begin{array}{r}
9 \pi r^{3} /\left(16 R^{3} \alpha^{4}\right) \quad: \alpha \geq r / R \\
\frac{3 r^{3}}{32 R^{3} \alpha^{4}}(12 \lambda-8 \sin (2 \lambda)+\sin (4 \lambda)) \\
: \sin \lambda=\alpha R / r \leq 1
\end{array}\right.
\end{aligned}
$$

This small angle approximation to $S(\alpha, r)$ should be reasonable for studying at least the first two peaks of $g_{v}(r)$, given that experimentally the radius of the fireball in central heavy ion collisions is $\sim 6 \mathrm{fm}$ and the inter-nucleon separation in nuclear matter is $\sim 2 \mathrm{fm}$.

When the rotational symmetry is a good approximation, it is convenient to decompose the angular distribution $w(\alpha)$ in terms of the orthogonal Legendre polynomials:

$$
w(\alpha)=\sum_{l=0}^{\infty} C_{l}\left(\frac{2 l+1}{4 \pi}\right) P_{l}(\cos \alpha),
$$




$$
C_{l}=2 \pi \int_{-1}^{1} d(\cos \alpha) w(\alpha) P_{l}(\cos \alpha) .
$$

An estimate of $w(\alpha)$ can then be compared to experimental data using $\cos \alpha=\cos \theta \cos \theta^{\prime}+\sin \theta \sin \theta^{\prime} \cos \left(\phi-\phi^{\prime}\right)$, but $C_{l}$ cannot be extracted from the experimental data unless full angular coverage is available. With only axial symmetry and limited angular coverage present, $w(\alpha)$ needs to be decomposed in terms of parity and azimuthal Fourier components as in Eqs.(15]16). Specifically, that avoids systematic errors in the construction of the connected distribution $w_{c}(\alpha)$. The addition theorem for the associated Legendre polynomials,

$P_{l}(\cos \alpha)=\sum_{m=-l}^{l}(-1)^{m} P_{l}^{m}(\cos \theta) P_{l}^{-m}\left(\cos \theta^{\prime}\right) e^{i m\left(\phi-\phi^{\prime}\right)}$,

then relates the coefficient functions according to

$$
\begin{aligned}
C_{m}^{\sigma}\left(\theta, \theta^{\prime}\right) & =(-1)^{m} \\
& \times \sum_{l=|m|}^{\infty} C_{l}\left(\frac{2 l+1}{2}\right) P_{l}^{m}(\cos \theta) P_{l}^{-m}\left(\cos \theta^{\prime}\right),
\end{aligned}
$$

where the sum is restricted to even(odd) values of $l-|m|$ for $\sigma=+(-)$. The functions $C_{m}^{\sigma}\left(\theta, \theta^{\prime}\right)$ can be extracted from theoretical models as well as from the experimental data, and they can be restricted to suitable ranges of $|m|$ and $\theta$ depending on the resolution available.

There still remain gaps between the theoretical formalism described here and the actual experimental data. The most prominent one is the fact that the detectors record only the charged hadrons. Thus protons and antiprotons are observed but neutrons and anti-neutrons are not. The requisite baryon number correlations can be extracted from the data only if the observed subset of (anti)protons provides a faithful characterization of the total baryon number distribution - ideally the two should be proportional. Moreover, corrections need to be estimated due to only approximate equilibration of the fireball, non-uniformity of the QGP caused by the elliptic flow, and baryon number diffusion subsequent to hadronization, all of which are likely to weaken the oscillatory correlation signal. On the other hand, development of the hard baryon core during hadronization would enhance the oscillatory signal, compared to the softer QGP state at higher temperature. Despite these gaps, it is worthwhile to look for the two-point baryon number correlations in the experimental data, as a characteristic signature of the deconfinement phase transition. Specifically, experimental determination of the contrast between $g_{|v|}$ and $g_{v}$, and their departure from $g(r)=1$ corresponding to no correlations, would provide a modelindependent characterization of the baryon-antibaryon correlations.

\section{SUMMARY AND OUTLOOK}

The Polyakov loop is a widely used order parameter for understanding the finite temperature deconfinement phase transition in a gauge theory. Its dual description in terms of the flux tube model has the advantage that the gauge theory dynamics can be visualized in position space. This picture helps in connecting experimentally observable baryon number correlations with properties of the deconfinement phase transition. There is no fundamental interaction associated with the baryon number. So the baryon number correlations have to arise from its precursors in the QGP, i.e. the pattern of the flux tube vertices expected from QCD.

The experimental effort in studying heavy ion collisions has so far largely focused on single particle distributions. In this article, I have gone beyond that towards analysing multi-particle distributions. I have described how to extract the two-point baryon number correlations from the experimental data, and have also predicted that it would contain an oscillatory signal, based on theoretically expected QGP properties at the chemical freeze-out stage. The experimental data for moderate $p_{T}$ baryons in central heavy ion collisions are the best suited for observation of the predicted signal. The features to be quantified are the distance scale and the amplitude of the oscillatory correlations. The former is expected to be the inter-baryon separation $(\sim 2 \mathrm{fm})$, while the latter though uncertain at present would tell us a lot about how tightly or softly the QGP is packed. It is worth keeping in mind that the intense search for two-point correlations in the CMBR, theoretically expected but without any accurate prediction of its magnitude (inflation is a much more flexible theory than QCD), found the correlations at the level of $\Delta T \approx 10^{-5} T$, and provided a major boost to our understanding of cosmology.

As a different application, the flux tube picture may also help in improving the baryon production estimates of hadronization models, by replacing the creation of diquark pairs with the creation of baryonic vertex pairs. It can also be noted that the flux tube dynamics influences diffusion in the QGP. Specifically, formation of a flux tube network would suppress diffusion. Both diffusion and viscosity characterize transport properties of a fluid, incorporating dissipative dynamics and entropy production. The former is convenient to use in position space, the latter in momentum space, and the two can be related in a kinetic theory framework. Low diffusion is not incompatible with the small viscosity to entropy density ratio in the high entropy density QGP. Quantitative analysis of diffusion in the QGP requires a hydrodynamic framework, however, which is beyond the scope of the formalism discussed here. 
[1] M. Alford, A. Schmitt, K. Rajagopal and T. Schäfer, Rev. Mod. Phys. 80 (2008) 1455.

[2] D. Kharzeev, Proc. The 18th Particles and Nuclei International Conference, PANIC08, Eilat, Nucl. Phys. A 827 (2009) $118 \mathrm{c}$.

[3] L. McLerran, Proc. The Sixth International Conference on the Physics and Astrophysics of Quark Gluon Plasma, Goa, Nucl. Phys. A 862-863 (2011) 251.

[4] C. Loizides, Proc. 23rd Rencontres de Blois, Particle Physics and Cosmology, Chateau de Blois, France, May 2011 (unpublished)).

[5] S. Gupta, Proc. The XXVIIIth International Symposium on Lattice Field Theory, Villasimius, PoS (Lattice 2010) 007.

[6] K. Kanaya, Proc. The XXVIII International Symposium on Lattice Field Theory, Villasimius, PoS (Lattice 2010) 012 .

[7] U.W. Heinz, Proc. 2003 CERN-CLAF School of HighEnergy Physics, San Miguel Regla, Mexico, June 2003, ed. N. Ellis (CERN, Geneva, 2006), p.165, e-print arXiv: hep-ph/0407360.

[8] A. Patel, Nucl. Phys. B 243 (1984) 411.

[9] G. Ripka, Dual Superconductor Models of Color Confinement, Lecture Notes in Physics, Vol. 639, (Springer, Berlin, 2004).

[10] Many multi-quark hadron resonances are frequently described in terms of intermediate diquark components. The most attractive diquark configuration is the antisymmetric color-triplet one, which is covered by the $\epsilon_{a b c}$ structure of the baryonic vertex.

[11] The extension of the allowed values is fine for the bosonic variables $n_{i, \mu}$ and $v_{i}$, but it is not clear-cut for the fermionic variables $p_{i, f}$ due to the exclusion principle.

[12] A. Patel, Phys. Lett. 139B (1984) 394.

[13] A. Patel, Proc. International Conference on the Physics and Astrophysics of Quark-Gluon Plasma, Bombay, India, February 1988, ed. B. Sinha and S. Raha (World Scientific, Singapore, 1988), p.135.

[14] J. Condella and C. DeTar, Phys. Rev. D 61 (2000) 074023.

[15] M. Alford, S. Chandrasekharan, J. Cox and U.-J. Wiese, Nucl. Phys. B 602 (2001) 61.

[16] S. Kim, P. de Forcrand, S. Kratochvila and T. Takaishi, Proc. The XXIIIrd International Symposium on Lattice Field Theory, Dublin, PoS (LAT2005) 166.

[17] Y.D. Mercado, H.G. Evertz and C. Gattringer, Phys. Rev. Lett. 106 (2011) 222001.

[18] P.N. Meisinger and M.C. Ogilvie, Phys. Lett. B 379 (1996) 163.

[19] K. Fukushima, Phys. Lett. B 591 (2004) 277.

[20] C. Ratti, M.A. Thaler and W. Weise, Phys. Rev. D 73 (2006) 014019.

[21] See for instance, B. Abelev et al. (STAR Collaboration), Phys. Rev. C 79 (2009) 034909.

[22] The experiments take place at fixed baryon number, while theoretical results are easier to obtain at fixed chemical potential. The two descriptions are related by a Legendre transformation.

[23] S. Weinberg, Cosmology, (Oxford University Press, 2008).

[24] See for example, P.M. Chaikin and T.C. Lubensky, Principles of Condensed Matter Physics, (Cambridge University Press, 2000). 